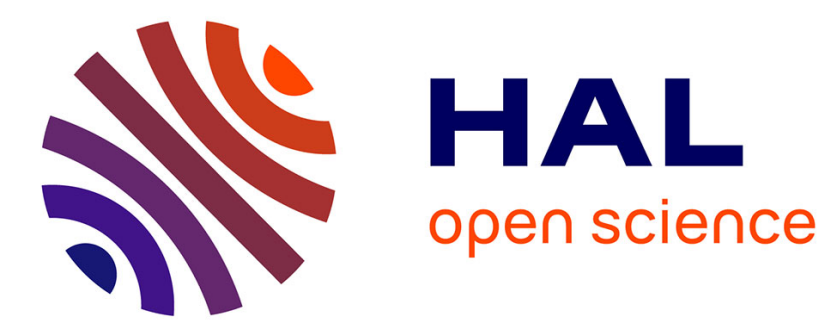

\title{
Calibration of a 3D working space by multilateration
} Martin Camboulives, Claire Lartigue, Pierre Bourdet, José Salgado

\section{To cite this version:}

Martin Camboulives, Claire Lartigue, Pierre Bourdet, José Salgado. Calibration of a 3D working space by multilateration. Precision Engineering, 2015, 10.1016/j.precisioneng.2015.11.005 - hal-01256972

\section{HAL Id: hal-01256972 \\ https://hal.science/hal-01256972}

Submitted on 15 Jan 2016

HAL is a multi-disciplinary open access archive for the deposit and dissemination of scientific research documents, whether they are published or not. The documents may come from teaching and research institutions in France or abroad, or from public or private research centers.
L'archive ouverte pluridisciplinaire HAL, est destinée au dépôt et à la diffusion de documents scientifiques de niveau recherche, publiés ou non, émanant des établissements d'enseignement et de recherche français ou étrangers, des laboratoires publics ou privés. 


\title{
Calibration of a 3-D working space by multilateration
}

\author{
Martin Camboulives $^{\mathrm{a}, \mathrm{b}}$, Claire Lartigue ${ }^{\mathrm{b}, *}$, Pierre Bourdet ${ }^{\mathrm{b}}$, José Salgado $^{\mathrm{a}}$ \\ ${ }^{a}$ LNE, 1 rue Gaston Boissier 75724 Paris cedex 15, France \\ ${ }^{b}$ LURPA, ENS de Cachan, 61 Avenue du Président Wilson F-94235 Cachan, France
}

\begin{abstract}
This paper deals with a calibration procedure of a 3D working space based on multilateration using a unique tracking interferometer. The key point of the procedure, detailed for a Cartesian CMM, is the definition of a reference measuring system built from the successive locations of a single tracer independently of the machine kinematics. Procedure limits are thus highlighted and can be classified into three types : limits of the devices (interferometer and measuring rule performances), limits linked to the use of a single tracer (reflector reorientation for visibility purposes), and limits linked to the algorithm. To evaluate associated uncertainties, a virtual measurement module has been developed which simulates a realistic 3D calibration and allows the study of the influence of each uncertainty component on the calibration procedure. The approach applied to the calibration of a virtual CMM proves that the simulation module is an efficient tool to investigate uncertainties associated with calibration procedures based on multilateration using a single tracer.

Keywords: Multilateration, CMM calibration, Tracer interferometer, Uncertainties, Volumetric errors
\end{abstract}

\section{Introduction}

Calibrating a working space consists in identifying a volumetric error at each point of the space. It is usually performed using tools such as ball-plates, ball-

\footnotetext{
*. Corresponding author. Tel. :+33140472986

Email address: lartigue@lurpa.ens-cachan.fr (Claire Lartigue)
} 
bars and the associated techniques [7], [2] [4] [1]. These methods are generally

5 time consuming. For instance, the calibration of a classical CMM (Coordinate Measuring Machine) takes about three days. Recent methods use multilateration to calibrate the considered working space reducing this time to a single day. Multilateration consists in determining the coordinates of a given point from the distances between this given point and $\mathrm{N}$ points which are in practice the centers of highly accurate tracking interferometers (Laser Tracer) (1). The most efficient way to proceed consists in using four laser tracers simultaneously [12]. However, the device is expensive and most laboratories can not afford four of them. Therefore, methods are developed based on a single Laser Tracer [8], [11], and are also applied to machine tools [5].

15 This paper deals with multilateration techniques for calibrating a CMM using a single Laser Tracer. The distances are defined as the relative measured lengths between the highly-accurate center of the Laser Tracer - that is set in several different positions - and a retro-reflector that is attached to the point to be measured. A reference measuring frame is built from the measurements. The calibration thus consists in calculating the difference between the coordinates indicated by the machine and the coordinates evaluated in the reference measuring space for each point of the working space. In practice, the working space is discretized in a rectangular grid of points.

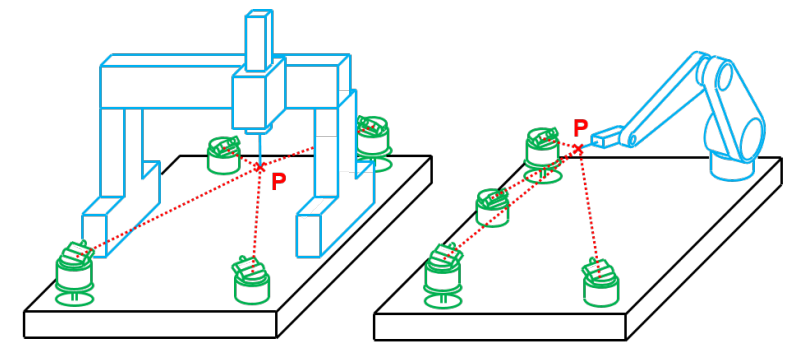

Machine of which the working space is calibrated

Reference measuring frame by the successive positions of the Laser Tracer

FIGURE 1: Superposition of a reference measuring frame and a working space. 
As a single Laser Tracer is used, the main difficulty lies in the construction at the extremities of the volume to be calibrated as recommended in [11]. To follow this recommendation, a specific strategy is proposed in the paper welladapted to CMM. Another contribution of our approach is the evaluation of uncertainties associated to the proposed procedure. Procedure limits are idento the use of a single interferometer, and limits linked to the algorithm used to calculate the reference frame. To evaluate uncertainties associated with the measurement, a virtual measurement module has been developed. Considering a working space with volumetric errors, the module simulates a realistic 3D caon the calibration procedure.

The rest of the paper is organized as follows. The next section details the construction of the reference measuring frame using a single Laser Tracer. Based on this, section 3 is dedicated to the calibration procedure applied to a CMM.

45 Section 4 focuses on the limits of the procedure and its associated uncertainties. The method to assess those uncertainties is also exposed. Section 5 concerns an application and the validation of our approach. Concluding remarks end the paper.

\section{Construction of a reference measuring system by multilateration}

Building the reference system requires that a set of points can be measured in the working space by all the positions of the Laser Tracer (see figure 2). The retro-reflector is located at each point of the discretized working space. For 
each position of the Laser Tracer, the length $d$ measured by the interferometer when the Laser Tracer aims at a point $P$ is recorded. During the procedure, $m$ successive positions of the Laser Tracer $L T_{j}$ aim at $N$ points $P_{i}$ in the working space. The unknowns are the coordinates of the Laser Tracer locations and the dead-path lengths $\left(x_{0 j}, y_{0 j}, z_{0 j}, d_{0 j}\right)$ and the three coordinates of each measured point $\left(x_{i}, y_{i}, z_{i}\right)$. For each point $P_{i}$, the following equations can be written :

$$
\left\|\overrightarrow{L T_{j} P_{i}}\right\|^{2}-\left(d_{i j}+d_{0 j}\right)^{2}=0
$$

Where dij is the length measured by the Laser Tracer in the position $L T_{j}$ aiming the point $P_{i}$, and $d_{0 j}$ is the death path associated with the position $L T_{j}$. Considering $j=1 . . m$ and $i=1 . . N$, this leads to a set of $N . m$ equations that are solved using a least square method. The unknowns are the 3 coordinates of the $m$ laser tracer positions, plus the $m$ number of associated death paths for each laser location, and plus the $3 . N$ coordinates of the points $P_{i}$ we want to localize. As the number of equations must be at least greater than the number of unknowns, the problem can be solved if :

$$
4 \cdot m+3 \cdot N \leq m \cdot N \Leftrightarrow N \geq \frac{4 \cdot m}{m-3}
$$

In order to obtain the point coordinates by multilateration, at least four lengths are required ( $m$ should be strictly greater than three), hence four positions of the Laser Tracer. According to equation (2) at least 16 points have to be measured to solve the problem. The reference measuring system is directly built from the successive positions of the Laser Tracer as shown in figure 2 . The Laser Tracer position $L T_{1}$ defines the origin $O$ of the frame. The positions ${ }_{75} L T_{1}, L T_{2}$, and $L T_{3}$ define the plane $(O, \vec{x}, \vec{y})$ for which the $\vec{x}$ axis is defined by considering the direction $L T_{1} L T_{2}$ and the $\vec{y}$ axis is normal to $\vec{x}$. Finally, the $\vec{z}$ axis is defined so that $(O, \vec{x}, \vec{y}, \vec{z})$ defines a direct orthonormal system. Then, as $x_{01}=y_{01}=z_{01}=0, y_{02}=z_{02}=0$ and $z_{03}=0$, this simplifies the problem 
by decreasing the number of unknowns by 6 . Considering equation (2), only 10 points are required to solve the problem. Point coordinates are finally obtained using equation (2), and are expressed in the reference measuring system $(O, \vec{x}, \vec{y}, \vec{z})$.

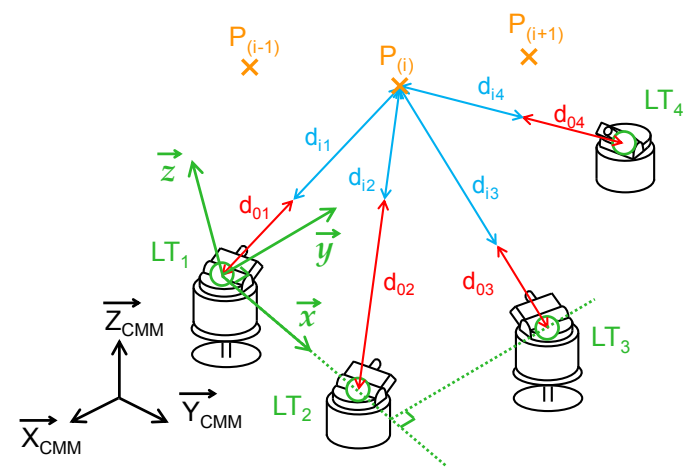

FIGURE 2: Construction of the reference measuring system.

Once the reference system is set,the coordinates of each point are obtained by multilateration in this system. Then the calibration procedure can be done 85 in several ways. The chosen one is detailed in section 3 .

\section{CMM Calibration}

Let us consider a $\mathrm{X} \rightarrow \mathrm{Y} \rightarrow \mathrm{Z}$ CMM. Mechanical defects in the guide ways generate kinematic errors (also referred as parametric errors). The most classical model to represent the parametric errors consists in defining six parametric errors on each axis, 3 rotational errors (i.e. pitch, roll and yaw) and 3 translational errors (i.e. the 2 straightnesses and the position error or trueness). The model is completed by considering the 3 squarenesses between each couple of axes. In this approach, it is chosen to identify each parameter one-by-one.

\subsection{Required trajectories to extract the parametric errors}

As rotations increase apparent straightnesses and position errors throughout the working space, rotations are determined first, then translational errors can 
be identified. Finally, squareness is evaluated thanks to an approach similar to that developed in [3].

In order to obtain the rotations, parallel trajectories must be set. For each the obtained volumetric errors, it is possible to retrieve the values of rotational errors [13]. Our approach is illustrated considering the Y-axis. The objective is to obtain the pitch yrx, the roll yry and the yaw yrz angles of the Y-axis (where the considered axis, the second letter is for translation or rotation, and the third concerns the axis of translation or rotation). In the case of yrx, the parallel trajectories are obtained thanks to two different configurations of the $\mathrm{Z}$-axis. For the yaw angle yrz, it is necessary to travel two trajectories parallel to the $\mathrm{Y}$-axis and offseted only in the $\mathrm{X}$-axis. As the $\mathrm{X}$-axis is before the $\mathrm{Y}$-axis for the CMM architecture considered in the paper, applying an offset on the retroreflector is necessary. Thus, the same trajectory is traveled twice : the first time with a neutral position of the retro-reflector and the second time with an offset in the $\vec{x}$ direction. This is illustrated in figure 3 . Note that the same happens for the Z-axis : it is necessary to offset the retro-reflector in the $\vec{x}$ and $\vec{y}$ directions in order to evaluate the rotations. On the contrary, no offset is required for the first axis as machine configurations are sufficient.

Equations 3 and 4 explain how to calculate the pitch yrx and the yaw yrz angles of the $\mathrm{Y}$-axis from the points obtained through the multilateration process, where $L$ is the offset in the $z$ direction and, $E_{x}, E_{y}$ and $E_{z}$ are the projections of the volumetric errors measured along the trajectories.

$$
\begin{aligned}
& \operatorname{yrx}(y)=\frac{E_{y}(x, y, z)-E_{y}(x, y, z+\Delta z)}{\Delta z} \\
& \operatorname{yrz}(y)=\frac{E_{y \text { offset }}(x, y, z)-E_{y}(x, y, z)}{L}
\end{aligned}
$$

When it comes to the roll angle yry,this angle can be calculated considering either the 2 parallel trajectories (shifted by $\Delta z$ ) or the trajectories with the offset $L$ on the $\mathrm{X}$-axis (see figure 4 ). Instead of choosing one or the other way 


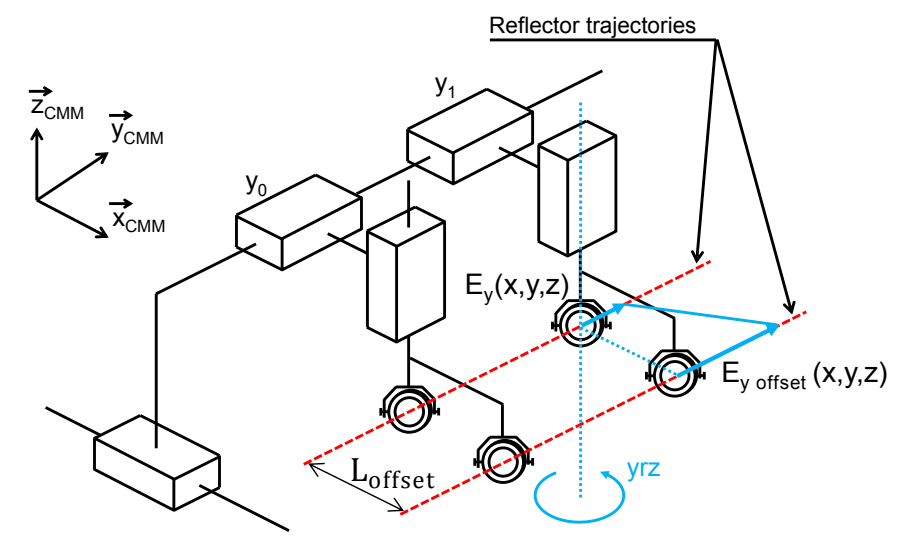

Figure 3: Offset in the $\vec{x}$ direction to observe the yaw yrz.

to calculate yry, we use the information redundancy to enhance the calculation reliability. However, a weighting is applied to give more importance to the measurement that presents the highest lever arm (5).

$$
\begin{aligned}
\operatorname{yry}(y)= & (1-\lambda) \cdot \frac{E_{x}(x, y, z+\Delta z)-E_{x}(x, y, z)}{\Delta z} \\
& +\lambda \cdot \frac{E_{z}(x, y, z)-E_{z \text { off } \mathrm{set}}(x, y, z)}{L}
\end{aligned}
$$

120

where $\lambda=\frac{L}{\Delta z}$. Then, if $\Delta z$ is very large compare to $L$, the importance is given to the first term. On the opposite, when $\Delta z$ is small compare to $L$, it is the second term which is the most important.

Once rotations are obtained, straightness and position errors are no longer affected by the machine configuration. They are measured along three straight trajectories aligned with the axis directions. Three trajectories are required for each axis calibration : from a reference trajectory, two others are obtained considering either the machine configuration (for the $\mathrm{X}$-axis rotations,yrx and yry) or an offset on the retro-reflector (for yrz and the Z-axis rotations). Figure 5 illustrates the complete procedure.

In practice, measurements are carried out according to the scheme displayed in figure 6. The Laser Tracer is set in one given position (A, B, C or D), and trajectories are traveled considering the three retro-reflector orientations ((a), 


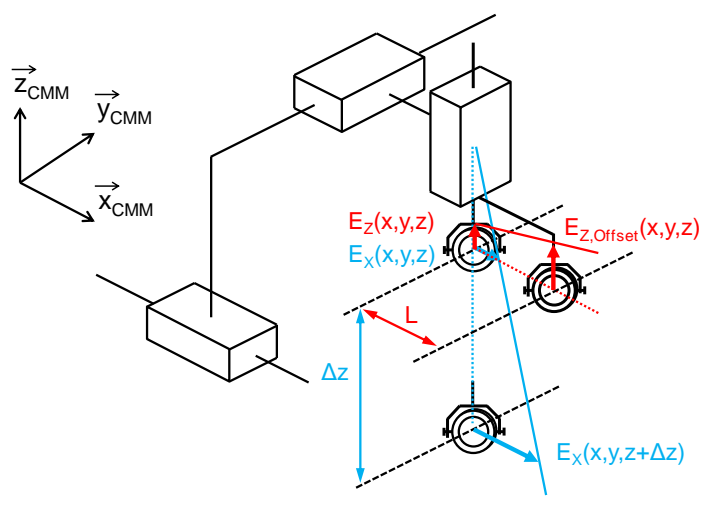

Figure 4: Retrieving of the roll yry thanks to errors in two directions.

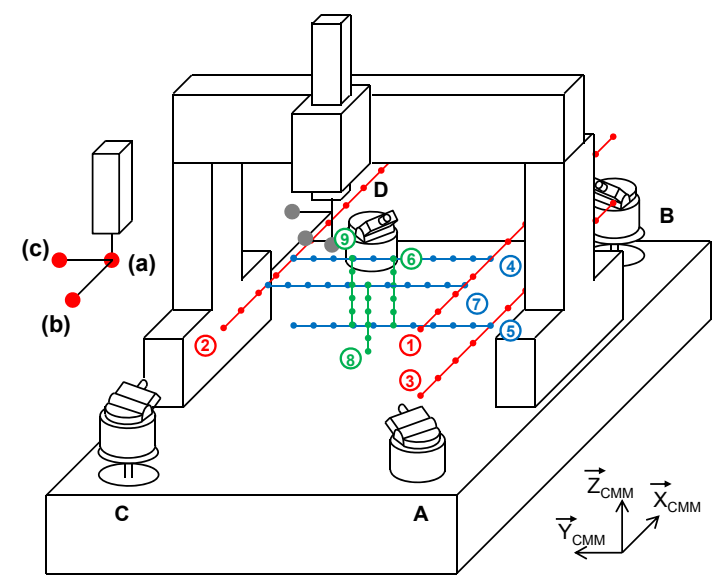

Figure 5: Trajectories and Laser Tracer positions to calibrate a 3-axis CMM. Trajectories (1) to (6) are proceeded with a neutral position of the retro-reflector noted (a). Trajectories (7) and (8) use the offset (b), and trajectory (9) uses the offset (c). 
(b), and (c)). Then, the Laser Tracer is moved to the next position, and the same procedure is repeated.It is assumed that, when the laser tracer is in position, there is no beam interruption during the whole measure, which is generally the case in practice.

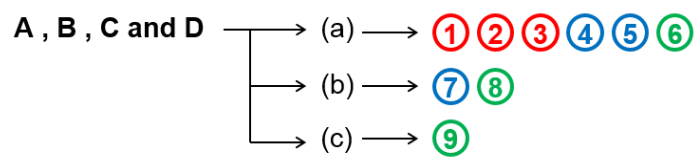

Figure 6: Measurement procedure for a 3-axis CMM.

\subsection{Calibration results for a 3-axis CMM in laboratory conditions}

The proposed approach, called M4LT (Multilateration using 4 Laser Tracer positions), has been applied to a portal type 3-axis CMM with a calibrated working space of approximately $900 \times 500 \times 400$ and a kinematic chain is $\mathrm{Y} \rightarrow \mathrm{X} \rightarrow \mathrm{Z}$. As mentioned above, the second axis requires an offset to determine its yaw ( $x r z$ in this case). The study thus refers to the parametric errors of $X$ as it illustrates all the ways to calculate parametric errors. In addition to the M4LT procedure, the Trac-Cal software developed by ETALON ${ }^{1}$ is also applied to the measurement data. Hence, calibration conditions are identical.

Uncertainties are calculated thanks to a Monte-Carlo method by only considering uncertainties linked to the measured lengths as it is proposed by Trac-Cal. Normally distributed numbers are added to the measured lengths with respect to the device characteristics. Results are displayed in figures 7 (for the translational errors) and 8 (for the rotational errors).

When using Trac-Cal, only the uncertainty associated with the interferometer, and the uncertainty of the retro-reflector offset measurement are considered. The computaton of the final uncertainties is not detailed in the software. In the figures, the blue lines represent the parametric errors yielded by Trac-Cal, and

1. www.etalon-ag.com 

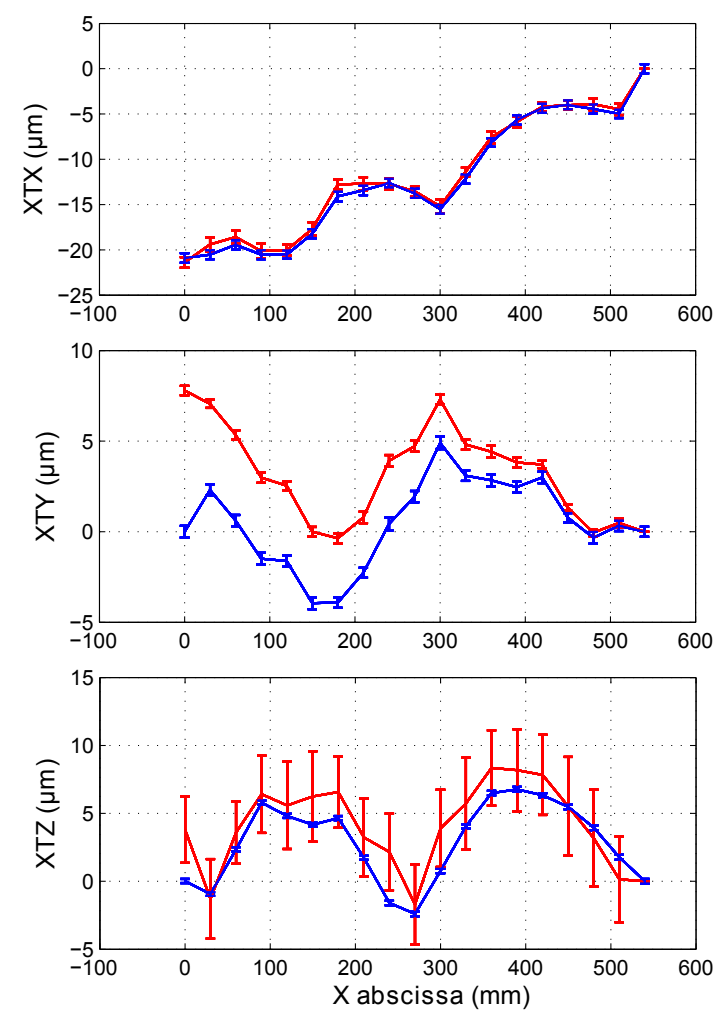

FiguRE 7: Translational errors of the second axis of the kinematic chain - parametric errors obtained with Trac-Cal (blue), parametric errors obtained using M4LT (red)

155 similar with both methods. Vertical straightness profiles are fairly close. The transversal straightness $x t y$ profiles are shifted by a constant slope that actually corresponds to the difference in squareness between the two approaches. Indeed, $x w y_{\text {Trac }-C a l} \approx-13.5 \mu \mathrm{rad}$ and $x w y_{M 4 L T} \approx-2.5 \mu \mathrm{rad}$. The $11 \mu \mathrm{rad}$ dif160 ference shows up in the slope between the two profiles. The pitch xry and roll xrx profiles are very similar to the ones yielded by Trac-Cal. On the opposite, yaw profiles reveal major differences all along the axis. Note that, for all the parametric errors, the error value is set at 0 at the abscissa $x=540 \mathrm{~mm}$ which is assumed to be the origin of the errors. 

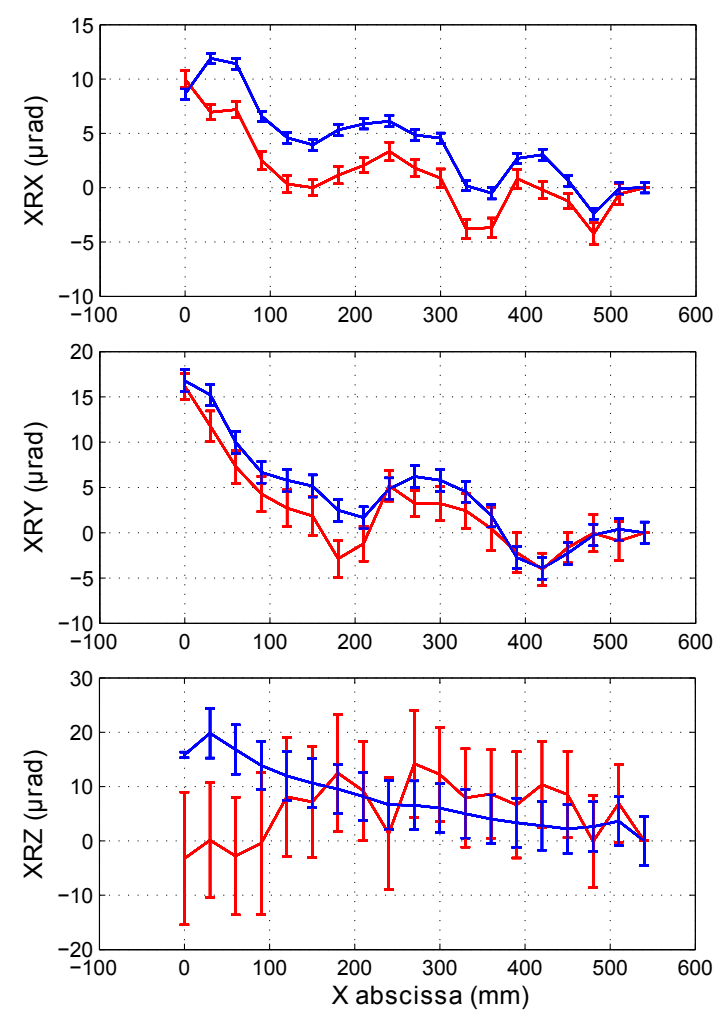

FIGURE 8: Rotational errors of the second axis of the kinematic chain - parametric errors obtained with Trac-Cal (blue), parametric errors obtained using M4LT (red)

Currently, uncertainty analysis is mainly based on the interferometric lengths [8] (see section 4.1). It is not clear whether other uncertainty components are taken into account, and how they are used in the process of calculating uncertainties. Therefore, we propose in the next section a precise analysis of all the sources of errors that contribute to the final uncertainty.

\section{Limits inherent to the method}

Each limit corresponds to a potential source of error involving errors or uncertainties on the calibration. 


\subsection{Interferometric length measurement}

When measuring a point $P_{i}$ from the Laser Tracer position $L T_{j}$, the Laser

Tracer interferometer delivers a length that corresponds to the absolute distance between the cat-eye and the Laser Tracer : $d_{i, j}+d_{0, j}$. Classically, the uncertainty model is linear [8] and the standard uncertainty $\sigma_{i, j}$ for each measured length is obtained thanks to the following equation :

$$
\begin{aligned}
& \sigma_{i, j}=u_{0}+\left(d_{i, j}+d_{0, j}\right) \cdot u_{L} \\
& \text { with : } \quad u_{0}=0.1 \mu \mathrm{m} \text { and } u_{L}=0.15 \mu \mathrm{m} / \mathrm{m}
\end{aligned}
$$

The enlarged uncertainty $U_{i j}$ is evaluated considering a coverage factor of 2 : $U_{i j}=2 . \sigma_{i, j}$.

\subsection{Machine positioning capabilities}

The machine that carries the retro-reflector is characterized by a limited positioning capability. It means that the position that is reached is not the programmed one. In the case of a CMM (which working volume is approximatively $\left.1 \mathrm{~m}^{3}\right)$, this positioning error is about $\pm 3 \mu \mathrm{m}$ and can be affected by parameters such as the feed rate or the direction of the displacement. Figure 9 illustrates the difference between the requested and the reached positions. As the procedure relies on the sequential multillateration using a single Laser Tracer, machine position errors affect the measured lengths but can be corrected, considering that volumetric errors can be assumed to be constant in a few micrometers cubic volume.

Let $\overrightarrow{n_{i j}}$ be the unit vector associated with the measurement direction. As $\left\|\overrightarrow{r_{i j}}\right\|$ vector is very small compared to the measured distances, the direction of $\overrightarrow{n_{i j}}$ is assumed to be hardly affected by the vector $\overrightarrow{i j}$. Hence, the positioning error can be projected onto the laser beam direction to correct the interferometric lengths :

$$
d_{i, j \text { corrected }}=d_{i, j}-\overrightarrow{n_{i j}} \cdot \overrightarrow{r_{i j}}
$$




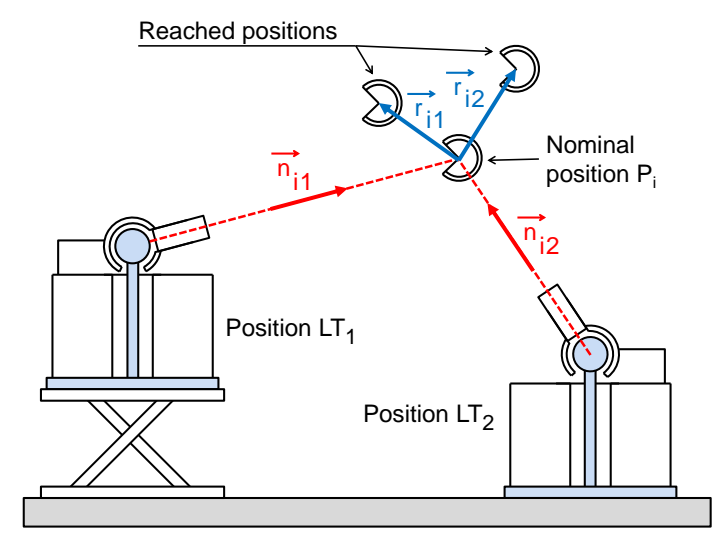

FiguRE 9: Correction of the carrier positioning error.

Finally,the CMM positioning capability can be characterized by the standard uncertainty $\sigma_{P o s . C M M}$, as $\sigma_{P o s . C M M, x}=\sigma_{P o s . C M M, y}=\sigma_{P o s . C M M, z}$ are assumed to be identical.

On the other hand, when reading the coordinates of the machine rules to express the vector $\overrightarrow{r_{i j}}$, position values are rounded to the rule resolution. Nowadays, most CMMs indicate axis positions with a resolution of about a tenth of a micrometer. Therefore, when correcting the machine positioning error thanks to equation 7 , the value of the vector $\overrightarrow{r_{i j}}$ is wrong by a value up to half of the rule resolution. The associated error is characterized by the standard uncertainty $\sigma_{\text {encoder }}$

\subsection{Retro-reflector reorientation}

Working with a single interferometer instead of four requires to repeat the same pattern of points four times in the working space with different positions of the Laser Tracer and different orientations of the retro-reflector. Indeed, the angle from which the retro-reflector is visible by the Laser Tracer is limited (from 120 to 160 degrees at best). Figure 10 illustrates this problem.

The way the cat-eye is mounted at the end of the kinematic chain is arbitrary. Hence, once the orientation is modified, the center of the cat-eye is moved by a few millimeters. Thus, the point targeted by the laser beam is basically not the 


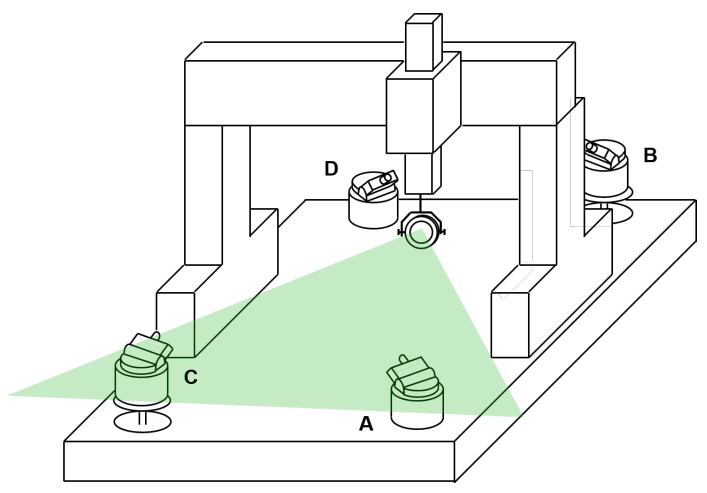

Figure 10: Visibility of the Cat-Eye in the case of a CMM calibration.

same for each position of the Laser Tracer. As mentioned previously, obtaining rotations sometimes requires to offset the retro-reflector. This offset can be performed by a rotation of the probe which is fairly accurate, or it can be realized using modular elements (see figure 11).

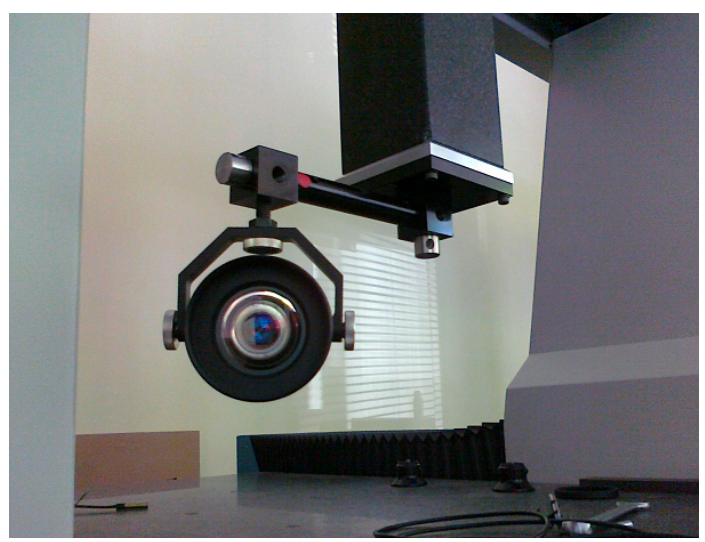

FIGURE 11: Use of modular elements to offset the reflector.

The difference between the actual offsets and the nominal one leads to uncertainties as this difference is affected by the rotations of the structure. Hence, a parasite error is measured which is only due to the reflector mounting.

Whatever the technology used to realize the offset, it is assumed that the 
cat-eye mounting error is normally distributed around a central offset value as illustrated in Figure 12.

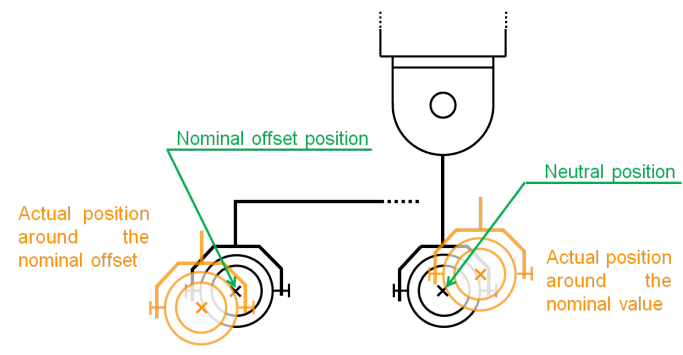

Figure 12: Dispersion of the cat-eye mounting (CEM) position around its desired position.

225

It is also assumed that $\sigma_{C E M, x}=\sigma_{C E M, y}=\sigma_{C E M, z}$, standard uncertainties of the components of the offset vectors, are identical. Therefore, the error association to the cat-eye mounting is characterized by a unique value $\sigma_{C E M}$.

\subsection{Optimization algorithm and initial step}

In order to solve the system of non-linear equations presented in section 2, an iterative algorithm is used. Most resolution methods rely on the gradient approach. Results can be affected by the choice of the initial solution which is necessarily given to achieve the calculation. However, a preliminary analysis showed that the influence of the algorithm is negligible relatively to other sources of errors. This source of errors will not be considered in the rest of the paper.

\subsection{Synthesis}

The present analysis highlights that the interferometer is not the only source of uncertainty associated with the calibration process by multilateration. Indeed, the study showed the following sources of errors :

240

- the interferometer, characterized by the standard uncertainty $\sigma_{i, j}$ given by equation 6 ,

- the CMM positioning capabilities characterized by $\sigma_{P o s . C M M}$,

- the linear encoder resolution, characterized by $\sigma_{\text {encoder }}$, 
- the cat-eye (retroflector) mounting, characterized by $\sigma_{C E M}$.

Uncertainties associated to the interferometer and to the rule resolution are generally well-known, whereas uncertainties linked to the CMM positioning and the cat-eye mounting result from experiments. Indeed, the CMM positioning strongly depends on the combination between the chosen feeding rate of the machine and the distance between each sampling point. These parameters solely depends on the measurement strategy. All these factors affect the evaluation of the interferometric lengths and the construction of the reference measuring system, and consequently the result of the CMM calibration. The importance of the associated uncertainties is assessed next.

\section{Uncertainty analysis}

In order to assess the impact of each uncertainty component on the global result, a simulation module has been developed to replicate the calibration procedure. This module allows the definition of virtual CMM based on Monte Carlo simulations that use the uncertainty components. This approach is similar to that proposed in [10] for the evaluation of CMM measurement uncertainty but with a different objective. Actually, our approach aims at assessing the calibration procedure of a CMM whereas the approach developed in [10] is more dedicated to the assessment of measurements performed using CMMs.

The simulation module requires a first calibration procedure according to the scheme described in section 3. Considering this initial measurement process, the tracking interferometer positions $L T_{j}$ and the dead-paths are evaluated in the machine coordinate system as well as the coordinate of the point positions $x_{i j, \text { rules }}, y_{i j, \text { rules }}$ and $z_{i j, \text { rules }}$ and read on each axis (see table 1 ). Note that, by comparing the positions reached to the programmed positions of the point trajectory, it is possible to evaluate the uncertainty component $\sigma_{P o \text { S.CMM }}$ and $\sigma_{\text {Pos.CMM }}$ associated to the CMM positioning capabilities and to the cat-eye mounting. 


\begin{tabular}{|c|c|c|c|c|}
\hline \multicolumn{4}{|l|}{ Position $L T_{j}$} \\
\hline$P_{i}$ & $x_{\text {ij,rules }}$ & $y_{\text {ij,rules }}$ & $z_{\text {ij,rules }}$ & $d_{\text {ij,interfero }}$ \\
\hline$P_{1}$ & $x_{1 j, \text { rules }}$ & $y_{1 j, \text { rules }}$ & $z_{1 j, \text { rules }}$ & $d_{1 j, \text { interfero }}$ \\
\hline$P_{2}$ & $x_{2 j, \text { rules }}$ & $y_{2 j, \text { rules }}$ & $z_{2 j, \text { rules }}$ & $d_{2 j, \text { interfero }}$ \\
\hline$\ldots$ & $\ldots$ & $\ldots$ & $\ldots$ & $\ldots$ \\
\hline$P_{N}$ & $x_{N j, \text { rules }}$ & $y_{N j, \text { rules }}$ & $z_{N j, \text { rules }}$ & $d_{N j, \text { interfero }}$ \\
\hline
\end{tabular}

TABLE 1: Data files available at the end of the measurement process

Therefore, the input data of the simulation module are the measurement files yielded by the tracking interferometer at the end of the calibration procedure, and the values of the uncertainty components aforementioned.

\subsection{Uncertainty assessment module}

Given these data, the simulation module consists of three main steps as displayed in figure 13. This module aims at simulating the behavior of an actual CMM during the calibration procedure. Multiple calibration procedures are performed thanks to Monte-Carlo simulations, each one leading to different parametric error profiles because of the uncertainty sources. From the differences between error profiles it is possible to identify standard deviations associated with each parametric error. To reach this objective, the first step is the simulation of the measurement process. For this calculation, the Laser Tracer positions are assumed to be the same throughout the whole simulation process. Starting from the result of a first measurement procedure according to the trajectories defined in section 3.1, a probability distribution is assigned to each measured length according to equation, and to each point position by introducing the combined effect of the other uncertainty components. The simulated measurement files obtained at the end of this step are used in the second step to calculate the Reference Measuring System (RMSys) as detailed in section 2. The last step is the multilateration stage to obtain the point positions from which both the volumetric errors and the parametric errors can be deduced. 
Monte-Carlo simulations, from step 1 to step 3, are conducted $N$ times.

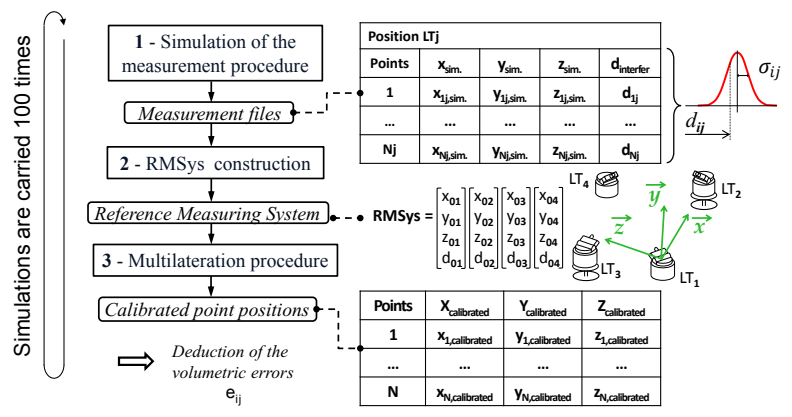

FiguRE 13: Steps of the simulation module.

295

uncertainty only is considered. The importance of taking into account all limits to assess the calibration procedure is clearly showed here. In particular, as expected, sequential multilateration using a single Laser Tracer set in 4 positions 


\begin{tabular}{|c|c|}
\hline Uncertainty components & Realistic values \\
\hline Interferometer & $u_{0}=0.1 \mu \mathrm{m}$ \\
$\sigma_{i, j}=\left[u_{0}+\left(d_{i, j}+d_{0, j}\right) \cdot u_{L}\right]$ & $u_{L}=0.15 \mu \mathrm{m} / \mathrm{m}$ \\
\hline CMM positioning & $\sigma_{\text {Pos } C M M}=1 \mu \mathrm{m}$ \\
\hline Linear encoder resolution & $\sigma_{\text {encoder }}=0.1 \mu \mathrm{m}$ \\
\hline Cat-eye mounting & $\sigma_{C E M}=3 \mathrm{~mm}$ \\
\hline
\end{tabular}

TABLE 2: Values of the uncertainty components for Monte-Carlo simulations.
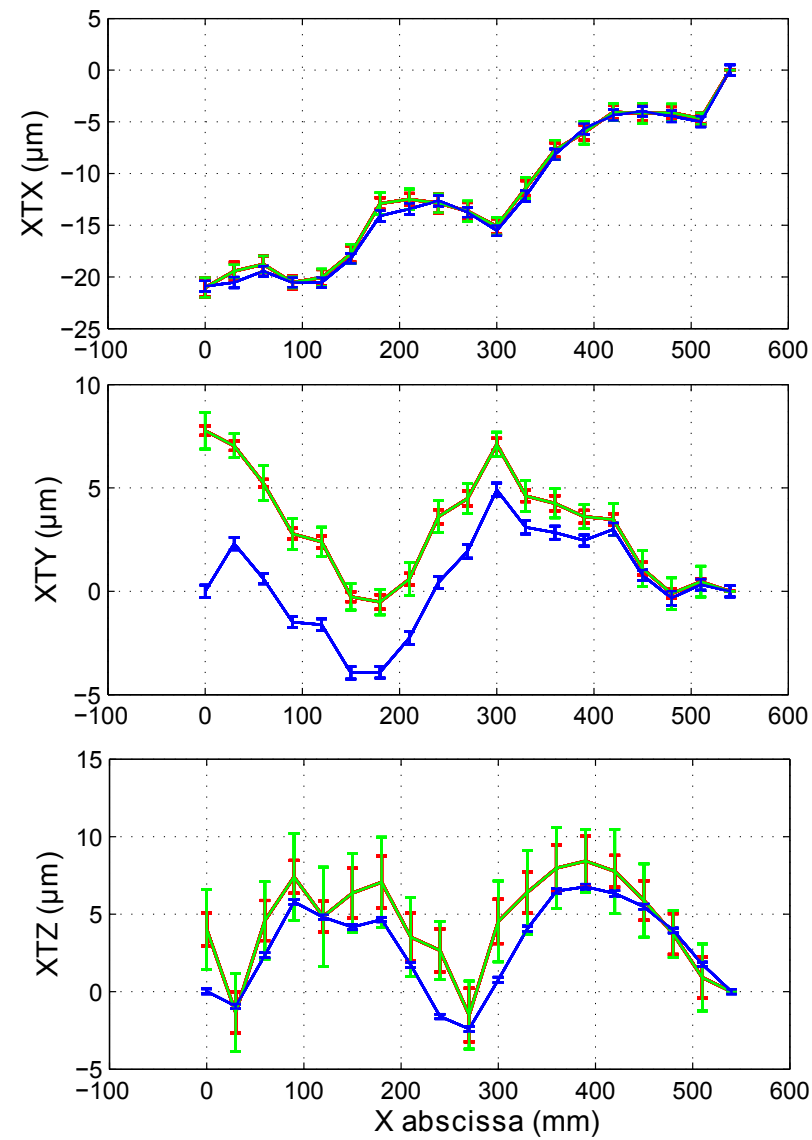

Figure 14: Translational errors for the second axis of the CMM - all uncertainty components (green) - the interferometer alone (red)- results obtained using Trac-Cal(blue) 
instead of using simultaneously 4 Laser Tracers (associated more specifically with the cat-eye mounting) is not influence free. Let us see now the relative influence of each uncertainty component in the global budget.
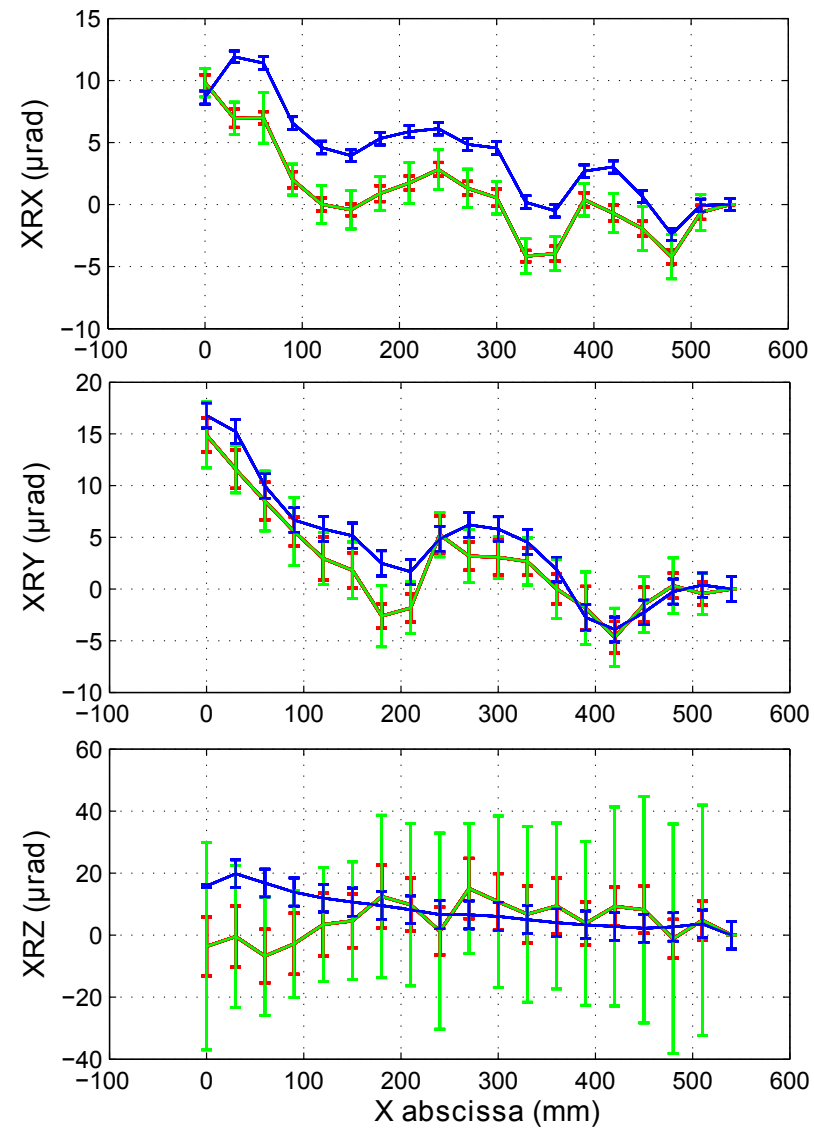

FiguRE 15: Rotational errors for the second axis of the CMM - all uncertainty components (green)- the interferometer alone (red)- results obtained using Trac-Cal(blue)

\subsection{Relative influence of each uncertainty component.}

To study the influence of each component, each one is investigated separately in the Monte-Carlo simulations. That is to say that the effect of the other components is eliminated when the importance of one is considered. In order to observe this relative influence, two indicators are defined. 
The first one is referred to as the RMSys quality indicator. It aims at assessing the importance of each uncertainty component on the construction of the Reference Measuring System. After the $N$ Monte Carlo simulations, a standard deviation $\sigma_{L T_{j}}$ can be assigned to each position $L T_{j}$ of the laser tracer. The $\sigma_{R M S y s}$ quality indicator is defined as the mean standard deviation :

$$
\sigma_{R M S y s}=\frac{1}{4} \cdot \sum_{j=1}^{4} \sigma_{L T_{j}}
$$

The second one is the mean value of the uncertainties associated to the parametric errors that do not require an offset of the retro-reflector. In the expression $9, u_{0}$ trans. $=1 \mu \mathrm{m}$ and $u_{0}$ rot. $=1 \mu \mathrm{rad}$ to obtain an expression without any dimension.

$$
\begin{aligned}
& \overline{u_{\text {param }, \text { direct }}}=\frac{1}{17} \cdot\left(\frac{u_{x t x, \text { moy }}+u_{x t y, \text { moy }}+u_{x t z, \text { moy }}}{u_{0 \text { trans. }}}+\right. \\
& \frac{u_{x r x, m o y}+u_{x r y, m o y}+u_{x r z, m o y}}{u_{0 \text { rot }}}+ \\
& \frac{u_{y t x, m o y}+u_{y t y, m o y}+u_{y t z, \text { moy }}}{u_{0 \text { trans. }}}+ \\
& \frac{u_{y r x, m o y}+u_{y r y, m o y}}{u_{0 \text { rot. }}}+ \\
& \frac{u_{z t x, \text { moy }}+u_{z t y, \text { moy }}+u_{z t z, \text { moy }}}{u_{0 \text { trans. }}}+ \\
& \left.\frac{u_{x w y}}{u_{0 \text { rot. }}}+\frac{u_{x w z}}{u_{0 \text { rot. }}}+\frac{u_{y w z}}{u_{0 \text { rot. }}}\right) \\
& \text { With for instance : } u_{x t x, \text { moy }}=\frac{\sum_{i=1}^{n_{X}} u_{x t x_{i}}}{n_{X}}
\end{aligned}
$$

For this second criterion, uncertainties on squareness have the same weight than a full error parameter profile. Indeed, an error on squareness involves a slope on straightness. Plus, the choice is made to only observe parameters that do not require an offset of the reflector. It is because uncertainties are much greater on the latter.

With the numerical values reported in table 2 , the influence of each parameter is displayed in Figure 16. 

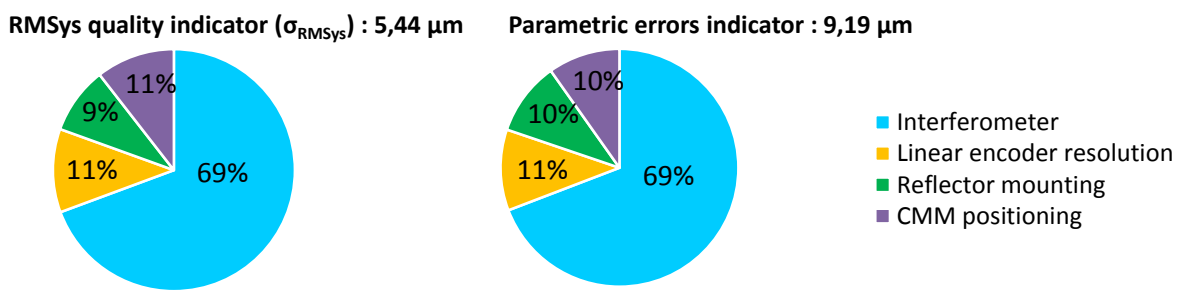

FiguRE 16: Influence of each component on the uncertainty budget

This figure highlights the importance of the interferometer in the global uncertainties, but also reveals that a third of the uncertainties comes from other error sources. Taking every component into account in the uncertainties budget is therefore a more reliable and relevant way to evaluate the quality of the considered CMM.It is important to notice that the effect of the CMM positioning associated to the use of 4 laser tracers has a limited influence. This is in particular due to the fact that each length $d_{i, j}$ is corrected according to equation 7 . Whereas it could be expected a major influence of the CMM positioning when using 4 laser tracer positions, this influence is compensated by a very small value of the rule resolution. This enhances the great importance of the correction of the measured length.

\section{Conclusion}

This paper presents an approach to determine volumetric errors of a working space using multilateration. Setting a reference measuring system built on the successive locations of a single tracer is the key point of this method. Indeed, it is independent from the architecture of the addressed machine. Only the lengths provided by the tracking interferometer are taken into account in the calculation, which is a benefit as inteferometric lenghts can be consider as references. Once the reference measuring frame is built, point coordinates are obtained by multilateration. Then calibrating the working space can be done either point by point or by extracting the kinematic chain parametric errors. The originality is 
that the limits of the method are explored. Procedure limits are identified highlighting the importance of the interferometer used for the measurement, but also the importance of the limit linked to the use of a unique interferometer. To evaluate uncertainties associated to these limits, a virtual measurement module is developed. Considering a working space with volumetric errors, the module simulates a realistic 3D calibration and allows the study of the influence of each uncertainty component on the calibration procedure thanks to a Monte-Carlo approach. In the paper, the method is applied to a CMM with efficiency. Parametric errors are determined along with associated uncertainties showing the relative importance of each limit. Future works will focus on the application of the approach to a 3D working space different from a CMM.

\section{Aknowledgements}

The authors want to thank Renishaw France for allowing the use of its experimental site for our experiments.

\section{References}

35 [1] Bourdet P. Contribution la mesure tridimensionnelle : Modle d'identification des surfaces, mtrologie fonctionnelle des pices mcaniques, correction gomtrique des machines mesurer tridimensionnelles. Thse d'Etat, Nancy I - LURPA ENS Cachan, 23 Juin 1987

[2] Evans C.J., Hocken R.J., Estler W.T. Self-calibration : reversal, redundancy, error separation and absolute testing Annals of the CIRP, $1996: 45(2): 617-634$

[3] Kruth J.P., Zhou L., Van den Bergh C., Vanherck P. A method for squareness error verification on a coordinate measuring machine. The International Journal of Advanced Manufacturing Technology, 2003 :21:874-878 
[4] Kunzmann H., Trapet F., Waeldele F. A uniform concept for calibration, acceptance test and periodic inspection of coordinate measuring machines using reference objects Annals of the CIRP, $1990: 39(1): 561-564$

[5] Linares J.-M., Chaves-Jacob J., Schwenke H., Longstaff A., Fletcher S., Flore J., Uhlmann E., Wintering J., Impact of measurement procedure when error mapping and compensating a small CNC machine using a multilateration laser interferometer Precision Engineering, 2014, 38 :578588.

[6] Hughes E.B.(NPL), Wilson A.(NPL), Peggs G.N.(NPL) Design of a highaccuracy CMM based on multilateration techniques Annals of the CIRP, $2000: 49(1): 391-394$

[7] Sartori S., Zhang G.X. Geometric error measurement and compensation of machines Annals of the CIRP, 1995 :44(2) :599-609

[8] Schwenke H.(PTB), Franke M.(PTB), Hannaford J.(NPL) Error mapping of CMMs and machine tools by a single tracking interferomter. Annals of the CIRP, $2005: 54(1): 475-478$

[9] Schwenke H., Knapp W., Haitjema H., Weckenmann A., Schmitt R., Delbressine F. Geometric error measurement and compensation of machines an update Annals of the CIRP, $2008: 57(2): 660-675$

[10] Sladek J., Gaska A. Evaluation of coordinate measurement uncertainty with the use of virtual model based on Monte Carlo Method Measurement, $2010: 45: 1564-1575$

[11] Umetsu K., Furutnani R., Osawa S., Takatsuji T., Kurosawa T., Geometric calibration of a coordinate measuring machine using a laser tracking system Measurement Science and Technology, 2005 :16:2466-2472

[12] Wendt K., Franke M., Haertig F. Measuring large 3D structures using four portable tracking laser interferometers Measurement, 2010 :45: 23392345 
[13] Trapet E., Waeldele F. Determination of the parametric errors of Coordinate measuring machines and machine tools using reference objects VDI Berichte Nr. 761, 1989 